\title{
FLUORESCENCE SPECTROSGOPY OF DERMAL WOUNDS IN RATS
}

\author{
Yuanlong Yang, ${ }^{\dagger}$ Lee David Mitchell, ${ }^{*}$ and R. R. Alfano ${ }^{\dagger}$ \\ ${ }^{\dagger}$ The City College and Graduate School of City University of New York, Institute for Ultrafast \\ Spectroscopy and Lasers, New York State Center for Advanced Technology for Ultrafast Photonic \\ Materials, Mediphotonics Laboratory, New York, NY 10031; ${ }^{\ddagger}$ City University of New York \\ Medical School, Sophie Davis School of Biomedical Education, Department of Physiology, New York, \\ NY 10031 \\ (Paper JBO-088 received Apr. 26, 1996; revised manuscript received Sep. 9, 1996; accepted for publication Oct. 23, 1996.)
}

\begin{abstract}
Fluorescence spectra of dermal lesions on 48 rats were investigated using excitation wavelengths of 275, 300, and $340 \mathrm{~nm}$. Emissions at 340 and $460 \mathrm{~nm}$ were measured in both the forequarter and hindquarter lesions as function of time after incision. Unlike the 460-nm emission, intensity at $340 \mathrm{~nm}$ increased with time and then saturated. Control studies on intact skin and lesions in dead rats failed to demonstrate any time-dependent changes at these wavelengths. It appears that the increase in the 340-nm intensity is due to changes in the tryptophan level, and may reflect accumulation of fibrinogen in the early wound healing process. $\odot 1997$ Society of Photo-Optical Instrumentation Engineers.
\end{abstract}

Keywords optical spectroscopy; fluorescence dermal wound; healing.

\section{INTRODUCTION}

The use of optical spectroscopy to observe and influence biological processes is of growing interest for many medical applications. Recently, optical spectroscopy has been used to detect physical and chemical changes in cells and tissues and distinguish cancerous tissues from healthy ones in various organs ${ }^{1-10}$ and in normal and atherosclerotic aorta. ${ }^{11-14}$ Various investigators have also reported that laser irradiation at low energies might accelerate wound healing in both humans and animal experimental models. ${ }^{15-19}$

In the work reported here, fluorescence spectroscopy was used to investigate initial changes in dermal wound healing. It is hoped that this methodology will lead to the development of noninvasive tools and procedures in the real time for clinical treatment and evaluation of wounds and burns.

\section{EXPERIMENTAL METHOD AND MATERIALS}

Spectroscopic measurements were performed using a Mediscience Technology Corp. CD Scan. This instrument is based on a Perkin-Elmer LS50 spectrophotometer with key software. A schematic optical layout is shown in Figure 1. The light source is a xenon flash lamp. The power of the excitation light source incident on the sample is about $1 / 2 \mu \mathrm{W}$. There are two monochrometers, one in the excitation beam path and the other in the emission beam

Address all correspondence to R. R. Alfano. E-mail: alfano@sci.sun.ccny.cuny.edu path. The excitation wavelengths are selected by adjusting the angle of the grating, which is controlled by a stepping motor, and an adjustable slit. The selected excitation beam is focused by a mirror into a bifurcated optical fiber bundle, with a small portion of the excitation beam reflected by a beam splitter into a reference photomultiplier. The output signal of the reference photomultiplier is used to correct the emission signal for variation in excitation intensity. The emission light is collected by the fiber bundle and is passed to the entrance slit of the emission monochrometer. The emission wavelength is selected by adjusting the angle of its grating, which is also controlled by a stepping motor. Only the emission light at the selected wavelength is passed into the signal photomultiplier for detection. The probe consisted of a UV transmitting quartz fiber bundle. The sample end of the probe is a 6-cm diameter circular aperture, recessed $2 \mathrm{~mm}$ from the end of its ferrule. The individual fibers in the probe are randomly split into two separate bundles, with $2 \times 6-\mathrm{cm}$ rectangular apertures to match the spectrometer optics. During measurements, the probe was positioned normal to the scanned region. Special software was used for data collection, processing, and plotting.

The fluorescence spectra from dermal lesions were obtained using 275,300 , and $340 \mathrm{~nm}$ as excitation wavelengths. The excitation intensity at 300 $\mathrm{nm}(340 \mathrm{~nm})$ was approximately $25 \%(80 \%)$ greater than the excitation intensity at $275 \mathrm{~nm}$; however, the spectrophotometer normalized the signal by the 


\section{FLUORESCENCE AND EXCITATION INSTRUMENT}

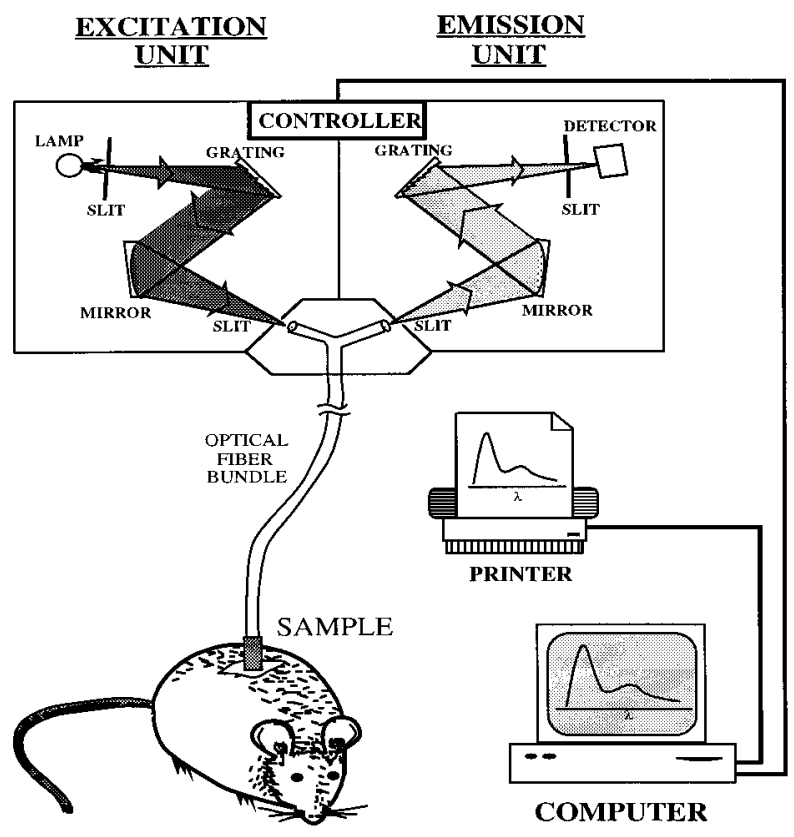

Fig. 1 Schematic diagram of fiber-based fluorescence measurement system.

excitation intensity. Two methods were employed to examine changes in the spectral response. (1) The whole spectrum was examined at 5-min intervals over a 2-h period. (2) The intensity of nine emission wavelengths was measured at 1-min intervals up to $150 \mathrm{~min}$ after incision. In addition the in vitro responses of separated blood plasma and various photoresponsive bioactive molecules in $0.9 \%$ sodium chloride solution were measured and the measured wavelengths are displayed in Table 1.

Table 1 Measured excitation and emission wavelengths from selected biological molecules.

\begin{tabular}{lcc}
\hline Excitation $(\mathrm{nm})$ & Emission $(\mathrm{nm})$ & Origin \\
\hline 275 & 310 & Collagen III \\
275 & 340 & Tryptophan \\
275 & 395 & Collagen II \\
300 & 340 & Tryptophan \\
300 & 375 & Pyridoxal 5'-phosphate \\
300 & 435 & Pyridoxic acid \\
340 & 390 & Collagen III \\
340 & 410 & Elastin \\
340 & 460 & NADH \\
\hline
\end{tabular}

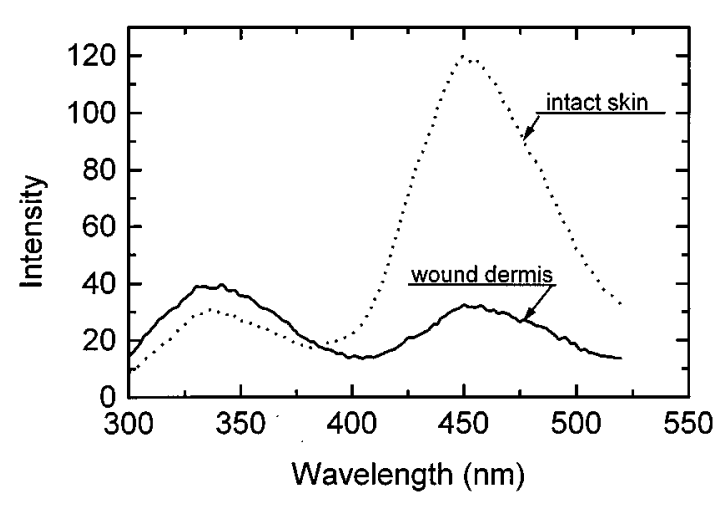

Fig. 2 Typical emission spectra of wound site and intact skin excited by $275 \mathrm{~nm}$.

Forty-eight 250 to $400-\mathrm{g}$ male Wistar rats were employed in the in vivo study. This study consisted of six experimental groups. The first group had a single lesion placed in either the right or left forequarter. Following the collection of data from the first lesion, the second group had an additional lesion placed on the opposite forequarter. The third group had a single lesion placed in either hindquarter. The fourth had two lesions, one in the forequarter and one in the hindquarter. The fifth group were controls consisting of shaved rats without lesions. The sixth group were controls consisting of shaved rats with lesions that died during the course of the study.

To prevent movement during measurements, all animals were anesthetized with a $40-\mathrm{mg} / \mathrm{kg}$ body weight dose of sodium pentobarbital administered intraperitoneally. The area of the wound sites was first shaved and cleaned with sterile alcohol swabs and air dried. Then, taking advantage of the loose pelt of this species, 1-cm long lesions made with sterile scalpel blades were extended through the dermis about $0.5 \mathrm{~mm}$ deep but no further. During the course of data collection the anesthetized rat was placed in a prostrate position and the optical fiber bundle was placed proximal to the lesion or to the shaved intact skin. The time course was recorded after $t=0$ when the lesion was made.

For in vitro examinations, $5 \mathrm{ml}$ of femoral blood was extracted from each of 6 anesthetized rats. To prevent clotting, an equal volume of heparinized saline (50 units $/ 0.9 \% \mathrm{NaCl}$ ) was added to each sample. A tabletop clinical centrifuge spinning at $3000 \mathrm{rpm}$ for $20 \mathrm{~min}$ was then used to separate plasma from hematocrit. Four-milliliter quartz cuvettes were then filled with the separated plasma and the emission spectra determined in the standard manner.

\section{RESULTS}

Figure 2 shows a typical fluorescence spectrum for intact shaved skin and a wound. In the shaved intact skin, fluorescence spectra for excitation wave- 


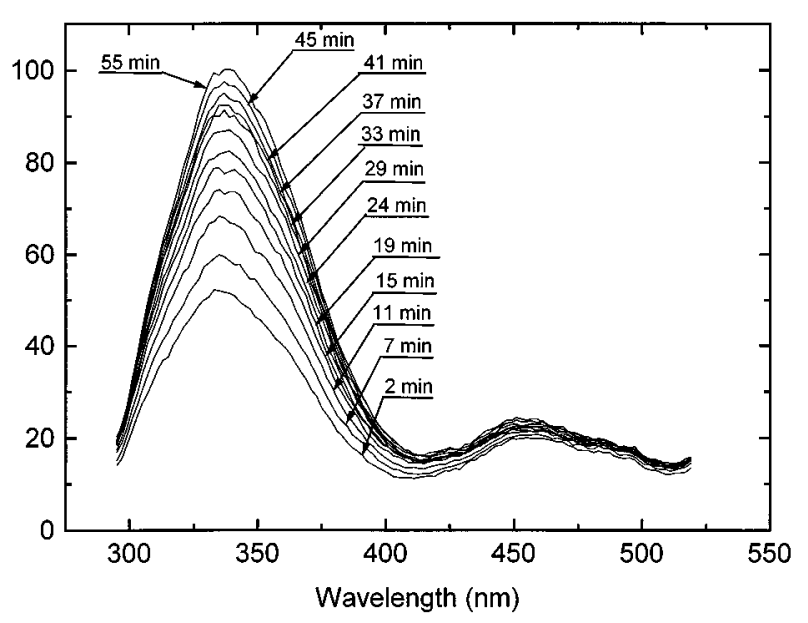

Fig. 3 The temporal behavior of the spectra of the wound site area after incision, excited by $275 \mathrm{~nm}$.

lengths 275 or $300 \mathrm{~nm}$ are similar. For these wavelengths, there are two emission intensity peaks; one is near $340 \mathrm{~nm}$ and the other is near $460 \mathrm{~nm}$. The amplitude of these two peaks varies with excitation wavelength. The dermal wound site also exhibited two peaks at 340 and $460 \mathrm{~nm}$. When a wound is compared with intact skin, the amplitudes of these peaks are quite different. The peak emission near $460 \mathrm{~nm}$ is markedly decreased and the peak emission at $340 \mathrm{~nm}$ is enhanced. When excited at either 300 or $275 \mathrm{~nm}$, both forequarter and hindquarter lesions exhibited emissions at 340 and near $460 \mathrm{~nm}$.

The most important observation was that in the wound site, the intensity of the $340 \mathrm{~nm}$ emission markedly increased with time after incision before finally reaching a plateau while that at $460 \mathrm{~nm}$ did not change in time (Figure 3). This observation at $340 \mathrm{~nm}$ was not dependent on the measuring methods (whether the whole spectrum was measured every $5 \mathrm{~min}$ or only the amplitude of the fluorescence spectrum was measured at 1-min intervals). The emission kinetics at $340 \mathrm{~nm}$ was in sharp contrast to the emission at near $460 \mathrm{~nm}$, which was unaffected by the passage of time (Figure 4) from the time after incision of the wound.

The analysis of the average rise time of 340-nm emissions for the first wound (Figure 5) was calculated using 15 rats with one forequarter lesion. The average rise time of a 340-nm emission for the second wound was calculated using 9 rats with forequarter lesions (Figure 5). Emission intensities were monitored at 1-min intervals. Rise-time curves were then constructed for each rat. The curves were then normalized and their combined means and standard deviations calculated. The average rise time of $90 \%$ maximum is $52.6 \pm 37 \mathrm{~min}$ for forequarter and $44 \pm 31 \mathrm{~min}$ for hindquarter. It was found that the second wound's pattern of spectral emissions did not statistically differ from that of the first. As in the first wound, the intensity of emission at $340 \mathrm{~nm}$

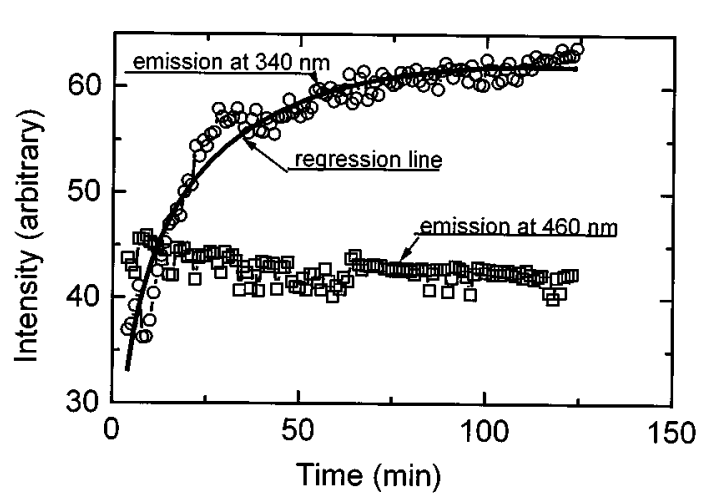

Fig. 4 The peak intensity from a wound area at $340 \mathrm{~nm}$ and near $460 \mathrm{~nm}$ as a function of time after incision. The sample is excited by 275 and $340 \mathrm{~nm}$, respectively.

increases with time while the intensity near $460 \mathrm{~nm}$ is unaltered.

To determine whether the observed emissions were due to the inadvertent selection of a unique portion of the skin, single lesions were made on the contralateral side in 6 rats and on the hindquarter in another group of 6 rats. Both contralateral and hindquarter lesions exhibited emissions at 340 and $460 \mathrm{~nm}$. The two curves appear to be similar and neither the order of the lesion nor its location altered the wound's rise time. In the control groups, emissions at $340 \mathrm{~nm}$ from intact skin and emissions from wounds in dead animals showed no alterations in intensities with time, as shown in Figure 6 for either 340- or 460-nm wavelengths.

The fluorescence peak at $340 \mathrm{~nm}$ excited by 275 $\mathrm{nm}$, and the peak at $460 \mathrm{~nm}$ excited by $340 \mathrm{~nm}$ is associated with tryptophan and reduced nicotinamide adenine dinucleotide (NADH) (Table 1), respectively. It is possible that the presence of tryptophan and NADH in dermal wounds is due to proteins leaking from the blood. Since whole blood does not respond to optical excitation, testing the above hypothesis required the separation of plasma from hematocrit. The emission spectra shown in

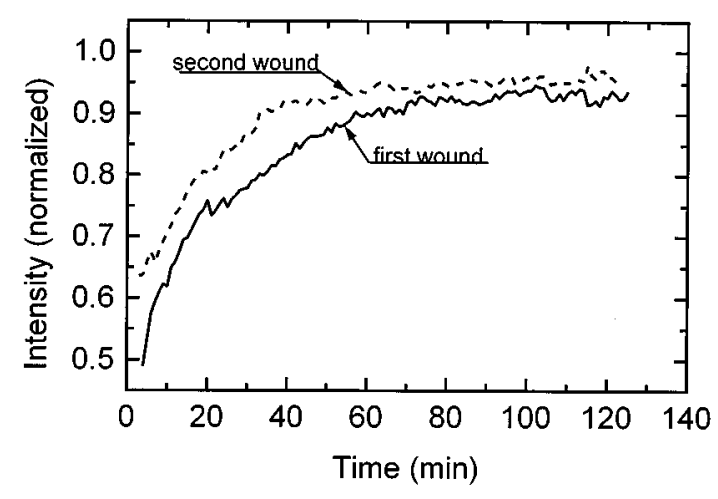

Fig. 5 Average intensity at $340 \mathrm{~nm}$ at first wound site area as a function of time ( $n=15$ rats) and average intensity of second wound area at $340 \mathrm{~nm}$ as a function of time ( $n=9$ rats). 


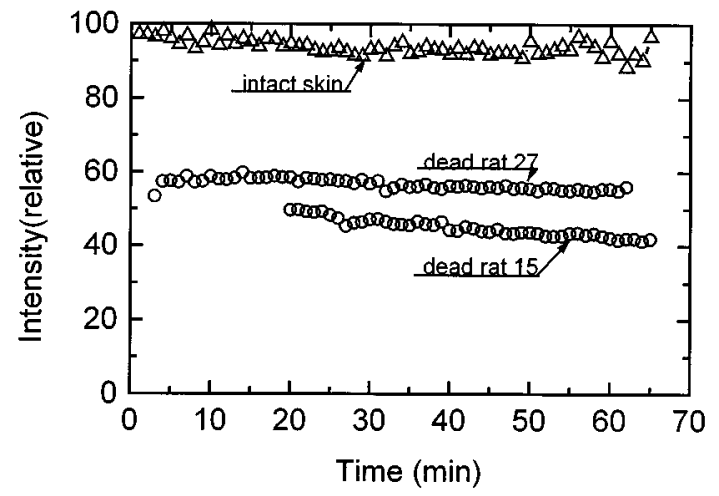

Fig. 6 Average intensity at 340-nm emission, excited by $275 \mathrm{~nm}$, for intact skin in live rats and lesions in dead rats, as a function of time.

Figure 7 confirm that tryptophan and NADH were found in the plasma samples. This suggests that the leakage of tryptophan into the wound increased while the NADH was unaltered with time. The hypothesis of leakage from the blood is not supported. Blood does not produce this level of signal.

It is also possible that the presence of tryptophan in the wound is due to the selective leaking of fibrin and fibrinogen from blood vessels. To test this concept, the in vitro spectrum of fibrin and fibrinogen in the solid state and dissolved in saline solution were measured. The spectra were nearly same for the two situations. Normalized spectra of fibrin and fibrinogen in saline and the wound area of rats are shown in Figures 8 and 9. When excited with 275 $\mathrm{nm}$, fibrin and fibrinogen emit with a peak near 340 $\mathrm{nm}$. The peak shift for fibrin is small. When excited with $340 \mathrm{~nm}$, the spectrum of fibrin emits a broader peak from 400 to $450 \mathrm{~nm}$. When excited with 275 $\mathrm{nm}$, however, fibrinogen emits a peak near $340 \mathrm{~nm}$ and a very small intensity at $460 \mathrm{~nm}$. The change in the spectrum emitted from fibrinogen would be closer to the emission spectrum of the wound area than that of fibrin.

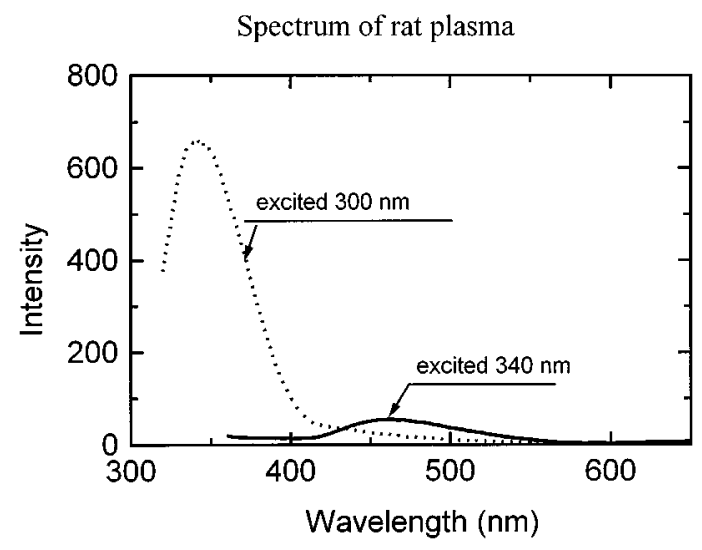

Fig. 7 Emission spectra of rat plasma excited by 300 and 340 $\mathrm{nm}$.

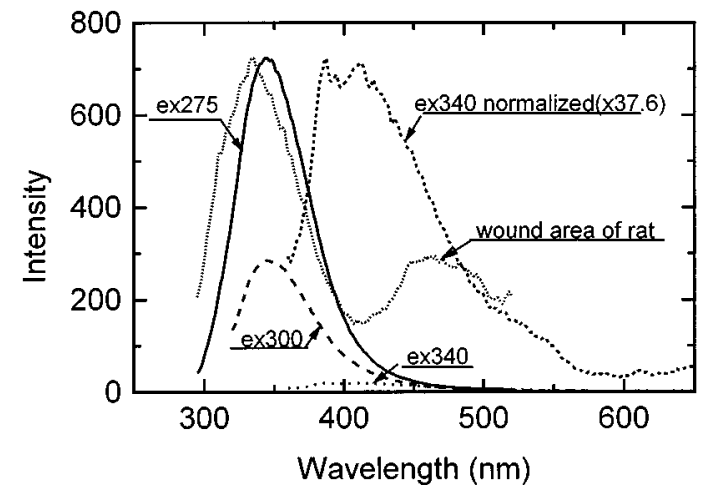

Fig. 8 Emission spectrum of the fibrin in saline excited by 275, 300 , and $340 \mathrm{~nm}$, and the spectrum of the wound area excited by $275 \mathrm{~nm}$ as reference. The normalized factor excited by $340 \mathrm{~nm}$ is 37.6 .

\section{DISCUSSION}

The significant observation was that when optically excited at 300 and $340 \mathrm{~nm}$, rat skin induces peak emissions at 340 and $460 \mathrm{~nm}$, respectively. The intensity from dermal wounds at $340 \mathrm{~nm}$ emissions grew, reaching $90 \%$ of its plateau value in $52.6 \mathrm{~min}$, which the $460 \mathrm{~nm}$ did not change after incision of a wound. Several questions arise from these observations. What produced the $340-\mathrm{nm}$ emission? Would the rising intensity of $340-\mathrm{nm}$ emissions occur in lesions on other areas of skin? Were the 340- and 460-nm emissions due to simple bleeding? Was the $340-\mathrm{nm}$ emission due to the lesion stimulating the release of a blood-borne hormonelike factor? Or was the 340-nm emission a sign of local, perhaps wound-healing processes? The initial observations were made on right forequarter lesions. The intensity of the 340-nm emission from the wounds increased with time. This is consistent with an accumulation of a tryptophan-like factor. To determine if this accumulation of a tryptophan-like factor was unique to right forequarter wounds, one and two

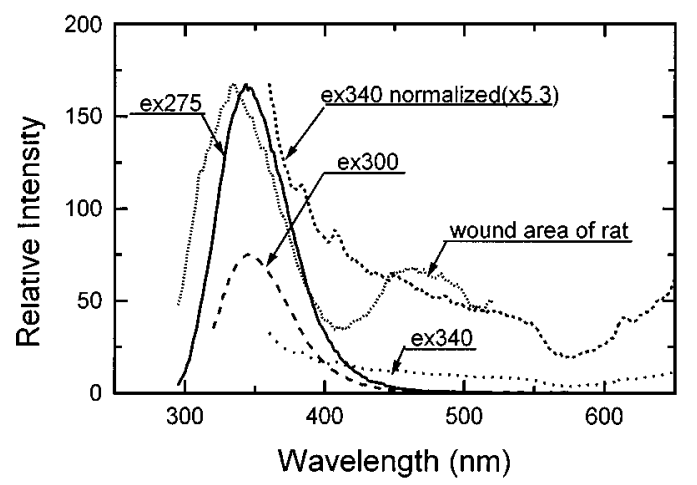

Fig. 9 Emission spectrum of the fibrinogen in saline excited by 275,300 , and $340 \mathrm{~nm}$, and the spectrum of wound area of a rat excited by $275 \mathrm{~nm}$ as reference. The normalized factor excited by $340 \mathrm{~nm}$ is 5.3 . 
transdermal lesions were made on other dorsal areas. All lesions emitted a similar spectrum.

It is possible that the tryptophanlike factor in dermal wounds is due to the stimulated release of a hormonelike blood-borne factor containing tryptophan. If such were the case, having already been stimulated, the tryptophanlike spectrum in a second wound would be expected to be more intense or at least to plateau earlier. To examine this hypothesis, the emission spectra of the second wounds were compared with those of the first. The second wound's pattern of spectral emissions did not differ from the first. The intensity of emission at $340 \mathrm{~nm}$ rose at the same apparent rate and intensity as in the first wound, while emission intensity at $460 \mathrm{~nm}$ was unaltered. These results suggest that whatever the origin of the 340-nm emission, it is most likely not due to hormonal action. It is likely that the $340-\mathrm{nm}$ emission is due to extravasation from unchanging blood stores or from dermal tissue.

A common observation was the accumulation of clear fluid in the wound. These observations suggested that the 340-nm emissions might be due to diapedesis from blood plasma. Since whole blood is spectrally inert, this hypothesis was examined by investigating the induced emission of blood plasma. When optically stimulated, plasma separated by centrifugation was found to generate emissions at 340 and $460 \mathrm{~nm}$. Furthermore, these emissions were unaltered with time. Since only the $340-\mathrm{nm}$ emission factor and not the 460-nm emission factor accumulated in the wound, the above observation supports the proposal that the source of wound tryptophanlike factor is not due to the unfiltered accumulation of blood plasma. Since neither shaved dermis nor dermal wounds in dead rats accumulated this tryptophanlike factor, it appears that the factor was not released from dermal stores. Since fibrin, when excited at $340 \mathrm{~nm}$ wavelength, emits a peak near $460 \mathrm{~nm}$, and fibrinogen does not, it appears most likely that the apparent accumulation of tryptophan is derived from fibrinogen. It appears that fibrinogen is a reasonable molecule for a built-up signal at $340 \mathrm{~nm}$ in a wound. When the dermis is damaged, the blood vessels in the region become selectively more permeable to fibrinogen. Our hypothesis that dermal wounds lead to a selective increase in fibrinogen permeability in the local vascular bed requires further study.

\section{Acknowledgment}

We thank Dr. H. Savage of the Sloan Kettering Cancer Center for help with the wound plasma work, Dr. A. Katz of the Mediphotonics Laboratory at The City College of New York for helpful suggestions, and New York State Technology Foundation and Mediscience Technology Corp. for support.

\section{REFERENCES}

1. R. R. Alfano and S. S. Yao, "Human teeth with and without caries studied by visible luminescence spectroscopy," $\mathrm{J}$. Dent. Res. 60, 120-122 (1981).

2. R. R. Alfano, D. B. Tata, J. Cordero, P. Tomashefsky, F. Longo, and M. A. Alfano, "Laser induced fluorescence spectroscopy from native cancerous and normal tissues," IEEE J. Quantum Electron. QE-20, 1507-1511 (1984).

3. R. R. Alfano, G. C. Tang, A. Pradhan, W. Lam, and D. S. Choy, "Fluorescence spectra from cancerous and normal human breast and lung tissue," IEEE J. Quantum Electron. QE-23, 1806-1811 (1987).

4. Wenlin Sha Glassman, C. H. Liu, G. C. Tang, S. Lubicz, and R. R. Alfano, "Ultraviolet excited fluorescence spectra from non-malignant and malignant tissue of the gynecological tract," Lasers Life Sci. 5(1-2), 49-58 (1991).

5. P. S. Anderson, E. Kjellin, E. Montan, K. Svanberg, and S. Svanberg, "Autofluorescence of various rodent tissues and human skin tumor samples," Lasers Med. Sci. 2, 41-49 (1987).

6. Y. Yuanlong, Y. Yanming, L. Fuming, L. Yufen, and M. Paozhong, "Characteristic autofluorescence for cancer diagnosis and its origin," Lasers Surg. Med 7, 528-532 (1987).

7. C. R. Kapadia, F. W. Cutruzzola, K. M. O’Brian, M. L. Stetz, R. Enriquez, and L. I. Deckelbaum, "Laser induced fluorescence spectroscopy on human colonic mucosa," Gastroenterology 99, 150-157 (1990).

8. R. M. Cothren, R. Richards-Kortum, M. V. Sivak, M. Fitzmaurice, R. P. Rava, G. A. Boyce, M. Doxtader, R. Blackman, T. B. Ivanc, B. Hayes, M. S. Feld, and R. E. Petras, "Gastrointestinal tissue diagnosis by laser-induced fluorescence spectroscopy at endoscopy," Gastrointest. Endosc. 36, 105111 (1990).

9. R. Richards-Kortum, R. P. Rava, R. E. Petras, M. Fitzmaurice, M. Sivak, and M. S. Feld, "Spectroscopy diagnosis of colonic dysplasia," Photochem. Photobiol. 50, 777-786 (1991).

10. K. T. Schomacker, J. K. Frisoli, C. C. Compton, T. J. Flotte, J. M. Richter, N. S. Nishioka, and T. F. Deutsch, “Ultraviolet laser induced fluorescence of colonic tissue: basic biology and diagnostic potential," Lasers Surg. Med. 12, 63-78 (1992).

11. P. S. Anderson, A. Gustafson, U. Stenram, K. Svanberg, and S. Svanberg, "Diagnosis of arterial atherosclerosis using laser-induced fluorescence," Lasers Med. Sci. 2, 261-266 (1987).

12. L. I. Deckelbaum, J. K. Lam, H. S. Cabin, K. S. Clubb, and M. B. Long, "Discrimination of normal and atherosclerotic aorta by laser-induced fluorescence," Lasers Surg. Med. 7, 330-335 (1987).

13. R. Richards-Kortum, R. P. Rava, R. Cothren, A. Metha, M. Fitzmaurice, N. B. Ratliff, J. R. Kramer, C. Kittrell, and M. S. Feld, "A model for extraction of diagnostic information from laser-induced fluorescence spectra of human artery wall," Spectrochem. Acta 45A, 87-93 (1989).

14. E. J. Gaffney, R. H. Clarke, A. R. Lucas, and J. M. Isner, "Correlation of fluorescence emission with the plaque content and intimal thickness of atherosclerotic coronary arteries," Laser Surg. Med. 9, 215-228 (1989).

15. E. Mester and E. Jaszagi-Nagy, "The effect of laser radiation on wound healing and collagen synthesis," Studia Biophys. 35, 227-230 (1973).

16. E. Mester, A. F. Mester, and A. Mester, "The biomedical effects of laser application," Lasers Surg. Med. 5, 31-39 (1985).

17. T. S. Lam, R. P. Abergel, J. C. Castel, R. M. Dwyer, and J. Uitto, "Laser stimulation of collagen synthesis in human skin fibroblast cultures," Lasers Life Sci. 1, 61-77 (1986).

18. F. H. M. Jongsma, A. E. J. M. vd Bogard, M. J. C. Van Gemert, and J. P. Hulsbergen Henning, "Is closure of open skin wounds in rats accelerated by argon laser exposure?" Lasers Surg. Med. 3, 75-80 (1983).

19. J. Hunter, K. Leonard, R. Wilson, G. Snider, and J. Divon, "Effects of low energy laser on wound healing in a porcine model," Lasers Surg. Med. 3, 285-290 (1984). 